so gibt es nach E.Strömgre ${ }^{8}$ eine maximale Bahngeschwindigkeit bei

$$
r=b \sqrt{2^{*}},
$$

während die Geschwindigkeiten im Zentrum und im Unendlichen Null sind. Im Gegensatz hierzu ist es nach W.Stepan off ${ }^{9}$ nicht möglich, einen Kugelsternhaufen nur aus Sternen aufzubauen, die sich in Pendelbahnen durch das Zentrum bewegen.

Aus (6) berechnet sich mit dem schätzungsweise bei $r=2,5 p c$ liegenden Maximum $b=1,8 p c$,

* S t r ö m g r e n gibt $r=\sqrt{6}$, da er von vornherein das spezielle Modell mit $b=\sqrt{\overline{3}}$ betrachtet.

8 Über Bewegungsformen in Globular Clusters. Astron. Nachr. 203, 17 [1916].

- Zur Frage über stationäre kugelförmige Sternhaufen. Russ. Astron. J. V, Nr. 2/3, 132 [1928]. während eine direkte Darstellung der Green s te in schen Zählungen nach (5) zu $b=1,55 p c$ führt. Durch diese hinreichende Übereinstimmung ist auf jeden Fall die Größenordnung gewahrt. Bedenkt man nun noch, daß unter Beibehaltung des Äquipartitionsprinzips der kinetischen Energie der Sterne des Haufens die Sterne kleinerer Masse plausiblerweise in der Zone maximaler Geschwindigkeiten häufiger vorkommen werden als in den übrigen Gebieten, so läßt sich der obige Befund auch dynamisch erklären. Die Einschränkung liegt nur darin, daß durch die Annahme von Kreisbahnen die Maxwellsche Geschwindigkeitsverteilung des isothermen Sterngases verletzt wird. Sie ist indessen weniger streng, wenn kreisähnliche Rosettenbahnen mit in die Betrachtung einbezogen werden.

\title{
Der mathematische Rahmen der Quantentheorie der Wellenfelder
}

\author{
Von Werner Heisenberg \\ Aus dem Max-P lanck - Institut für Physik, Göttingen \\ (Z. Naturforschg. 1, 608-622 [1946]; eingegangen am 5..Aug. 1946)
}

\begin{abstract}
Die übliche Quantentheorie der Wellenfelder, bei der man von einer H a m i l t o n Eunktion in Abhängigkeit von irgendwelchen Feldgrößen ausgeht, führt im allgemeinen zu Divergenzen. Der vorliegende Aufsatz stellt einen zusammenfassenden Bericht über verschiedene Arbeiten dar, die dureh Erweiterung des bisherigen Verfahrens den mathematischen Rahmen einer zukünftigen Theorie der Wellenfelder oder der Elementarteilchen festzulegen suchen. Dabei wird einerseits auf die Bedeutung einer unitären Matrix, der sogenannten Streumatrix, und einer mit ihr verknüpften hermiteschen Matrix hingewiesen; andererseits wird gezeigt, daß auch bei einer Erweiterung der bisherigen Wellengleichungen $\mathrm{zu}$ sehr allgemeinen Integro-Differentialgleichungen die Forderungen der Quantentheorie zu einem eindeutigen mathematischen Formalismus führen.
\end{abstract}

$\mathrm{D}^{\mathrm{i}}$ Quantentheorie der Wellenfelder in ihrer üblichen Form ist unbefriedigend, weil sie meist zu Divergenzen führt, die die strenge mathematische Behandlung der betreffenden Probleme verhindern. Eine Theorie dieser Art kann also nur als eine ausgearbeitete korrespondenzmäßige Behandlung der gestellten Fragen angesehen werden. Trotz der Erfolge bei der Quantenelektrodynámik ${ }^{1}$ oder der Theorie des Mesons ${ }^{2}$ ist der bisherige Formalismus also sicher noch nicht

1 Vergl. z. B. G. W en z e l, Z. Physik 86, 479; 86, $635 ; 87,726$ [1934] ; P. A. M. D i r a c, Proc. Roy. Soc. [London] 167, 148 [1938] und Bakerian lecture 1942 (?); F. B o p p, Ann. Physik 38, 345 [1940]; 42, 573 [1942].

2 S. Y u k a wa, Proc. physic.-math. Soc. Japan 17, 48 [1935]; H. F r ö h l i c h, W. H e i t l e r u. N. K e m$\mathrm{mer}$, Proc. Roy. Soc. [London] 166, 154 [1938]; richtig, und es entsteht die Frage, wie das strenge mathematische Schema für eine zukünftige Theorie der Elementarteilchen aussehen wird. Der Verfasser hat in den vergangenen Jahren in vier Arbeiten, die für die Zeitschrift für Physik bestimmt waren $^{3}$, den mathematischen Rahmen einer solchen Theorie erörtert und die Bedingungen studiert, denen sie zu genügen hat. Von den genannten Arbeiten ist die vierte infolge der Zeitumstände noch nicht erschienen, und auch die dritte scheint nur wenigen Physikern zugänglich zu sein. Ferner

B h a bha, Nature [London] 141, 117 [1938]; G. We n t z e l, Helv. Phys. Acta 13, 269 [1940]; 14, 633 [1941]; Z. Physik 118, 277 [1941].

3 W. H e i s e n be r g, Z. Physik 120, 513 (I); 120 , 673 (II) [1943]; 123, (?) (III) [1945]; im folgenden als l. c. I, II, III zitiert. Teil IV wird später erscheinen. 
sind in der Zwischenzeit Arbeiten anderer Autoren über den gleichen Gegenstand veröffentlicht worden, und es erscheint deshalb berechtigt, im folgenden eine zusammenfassende Darstellung dieses Problemkreises zu geben.

In den üblichen Theorien werden Begriffe benutzt wie: Wellenfunktionen an einem bestimmten Punkt in Raum und Zeit, Lage eines Teilchens, Energiedichte usw. Es erscheint fraglich, ob all diese Begriffe in einer richtigen Theorie verwendet werden können, da die Naturgesetze wahrscheinlich eine universelle Konstante von der Dimension einer Länge und der Größenordnung $10^{-13} \mathrm{~cm}$ enthalten. Die Existenz dieser Konstantén wird Schwierigkeiten hervorrufen bei der Anwendung eines Begriffes, der mit einem bestimmten RaumZeit-Punkt verknüpft ist. Es scheint daher notwendig, eine kleinere Zahl von Begriffen auszuwählen, die durch diese Schwierigkeiten nicht berührt werden, und die deshalb auch ein Bestandteil der zukünftigen Theorie sein können. Um . solche Begriffe zu finden, wird man zweckmäßig fragen, welche Größen direkt gemessen werden können. Die wichtigsten beobachtbaren Größen scheinen die Energiewerte abgeschlossener Systeme und die Wahrscheinlichkeiten für Stoß, Absorption und Emission zu sein. Da die Größen der zuletzt genannten Art in der Quantentheorie mit dem asymptotischen Verhalten der Wellenfunktion bei großen relativen Abständen der Teilchen verknüpft sind, wird man also annehmen, daß die Energiewerte der diskreten stationären Zustände und das asymptotische Verhalten der Wellenfunktion Begriffe sind, die auch in der zukünftigen Theorie der Elementarteilchen ihre Rolle spielen werden. Damit entsteht die Frage nach einer geeigneten mathematischen Darstellung dieser beobachtbaren Größen.

\section{Mathematische Darstellung der beobachtbaren Größen}

a) In den folgenden Rechnungen soll der gewöhnliche Formalismus der Quantentheorie zugrunde gelegt werden. Wir nehmen an, daß die Wellenfunktion von den Impuls-Koordinaten $\mathfrak{f}$ der Teilchen abhängt, die zugehörigen kinetischen Energien der Teilchen seien $k_{i}^{0}$; für den Gesamtimpuls und die gesamte kinetische Energie soll

$$
\mathfrak{K}=\sum_{i} \mathfrak{k}_{i} ; \quad K_{0}=\sum_{i} k_{i}^{0}
$$

gesetzt werden. All diese Größen werden zweckmäßig in rationellen Einheiten gemessen, bei denen sie mit Potenzen von $\hbar$ und $c$ zu multiplizieren sind, bis sie die Dimension irgendeiner Potenz einer Länge haben. Die ebene Welle wird in diesen Einheiten einfach durch $e^{i \text { pr }}$ dargestellt.

Die Wellenfunktion, die zu einem bestimmten Zustand im kontinuierlichen Spektrum gehört, kann durch die Impulse $\mathfrak{f}_{i}{ }^{\prime}$ der einfallenden ebenen Wellen definiert werden. Da die Wellenfunktion selbst von den Impulsen $\mathfrak{f}_{i}{ }^{\prime \prime}$ abhängt, kann man, wie $M o ̈ l l e r^{4}$ vorgeschlagen hat, die Wellenfunktionen $\psi_{\mathfrak{p} i}^{\prime}\left(\mathfrak{f}_{i}^{\prime \prime}\right) \mathrm{zu}$ einer Matrix $\left(\hat{f}_{i}^{\prime \prime}|\psi| \mathfrak{k}_{i}^{\prime}\right) \mathrm{zu}-$ sammenfassen. Wenn die Teilchen keinerlei Wechselwirkung miteinander haben, gilt für diese Matrix einfach :

$$
\left(\mathfrak{k}_{i}^{\prime \prime}|\psi| \mathfrak{k}_{i}^{\prime}\right)=\left(\mathfrak{k}_{i}^{\prime \prime}|1| \mathfrak{k}_{i}^{\prime}\right)=\delta\left(\mathfrak{k}_{i}^{\prime \prime}, \mathfrak{k}_{i}^{\prime}\right),
$$

oder in der Matrix-Schreibweise

$$
\psi=1 \text {. }
$$

Wenn eine Wechselwirkung vorhanden ist, muß jedoch noch eine auslaufende Welle hinzugefügt werden, um eine Lösung der entsprechenden Schrödinger-Gleichung zu bekommen. Diese auslaufende Welle kann nach Dir a $\mathrm{c}^{5}$ dargestellt werden durch das Produkt einer Funktion $\operatorname{der} \boldsymbol{f}_{i}{ }^{\prime \prime}$ und einer anderen Funktion, die eine besondere Art von Singularität am Punkt $\mathfrak{K}^{\prime}=\Re^{\prime \prime}, K_{0}^{\prime}=K_{0}^{\prime \prime}$ hat. Für die Darstellung dieser letzteren singulären Funktion führt man zweckmäßig die $A b$ kürzungen ein

$$
\begin{gathered}
\delta_{+}(k)=\frac{1}{2 \pi} \int_{0}^{\infty} \mathrm{e}^{i k t} d t ; \delta_{-}(k)=\frac{1}{2 \pi} \int_{-\infty}^{0} \mathrm{e}^{i k t} d t \\
\delta_{+}(k)+\delta_{-}(k)=\delta_{(k)} \\
\delta_{-}(k)=\delta_{+}^{*}(k)=\delta_{+}(-k)
\end{gathered}
$$

Man kann leicht zeigen, daß das Produkt

$$
\delta_{+}\left(K_{0}^{\prime}-K_{0}^{\prime \prime}\right) \delta\left(\mathfrak{K}^{\prime}-\mathfrak{K}^{\prime \prime}\right) f\left(\mathfrak{k}_{i}^{\prime \prime}\right)
$$

eine auslaufende Welle darstellt. Die Funktionen $\delta_{+}(k)$ sind im wesentlichen den D i r a c schen Ausdrücken

$$
\pm \frac{1}{2 \pi i k}+\frac{1}{2} \delta(k)
$$

äquivalent.

\footnotetext{
4 Chr, Möller, Verh. kgl. Dän. Akad. Wiss. 23, Nr. 1 [1945]; 24, Nr. 19 [1946].

5 P. A. M. D i r a c, Z. Physik 44, 585 [1927].
} 
Das vollständige System der Wellenfunktionen im kontinuierlichen Spektrum kann dann durch

$\left(\mathfrak{k}_{i}^{\prime \prime}|\psi| \mathfrak{k}_{i}^{\prime}\right)=$

$\left(\mathfrak{k}_{i}^{\prime \prime}|1| \mathfrak{k}_{i}^{\prime}\right)+\delta_{+}\left(K_{0}^{\prime}-K_{0}^{\prime \prime}\right) \delta\left(\mathfrak{K}^{\prime}-\mathfrak{K}^{\prime \prime}\right)\left(\mathfrak{k}_{i}^{\prime \prime}|f| \mathfrak{k}_{i}^{\prime}\right)$

$=\left(\mathfrak{k}_{i}^{\prime \prime}|1| \mathfrak{k}_{i}^{\prime}\right)+\delta_{+}\left(K_{0}^{\prime}-K_{0}^{\prime \prime}\right)\left(\mathfrak{k}_{i}^{\prime \prime}|\boldsymbol{r}| \mathfrak{k}_{i}^{\prime}\right)$

dargestellt werden. Für das asymptotische Verhalten der Wellen sind nur die Werte von $\left.\left(\mathfrak{k}_{i}{ }^{\prime \prime}\right] r \mid \mathfrak{f}^{\prime}{ }^{\prime}\right)$ in der unmittelbaren Nachbarschaft des singulären Punktes $\Re^{\prime}=\Re^{\prime \prime}, K_{0}^{\prime}=K_{,}^{\prime \prime}$ wichtig, da für die auslaufenden Teilchen Gesamtenergie und Gesamtimpuls erhalten bleiben. Der Rest der Matrix bestimmt das Verhalten der Wellenfunktion bei kleineren Abständen der Teilchen. Es ist deshalb zweckmäßig, eine neue Matrix $R$ durch die Definition

$$
\left(\mathfrak{k}_{i}^{\prime \prime}|R| \mathfrak{k}_{i}^{\prime}\right)=\delta\left(K_{0}^{\prime}-K_{0}^{\prime \prime}\right)\left(\mathfrak{k}_{i}^{\prime \prime}|r| \mathfrak{k}_{i}^{\prime}\right)
$$

einzuführen, ferner eine andere Matrix $S$ durch:

$$
S=1+R \text {. }
$$

Diese Matrix $S$ (oder $R$ ) enthält, wie gezeigt werden soll, alle notwendigen Informationen über die Größen, die als beobachtbare Größen bezeichnet worden sind.

Es sei hier noch angemerkt, daß ein Produkt von der $\operatorname{Art} \delta(\mathfrak{f}) \delta\left(k^{\mathbf{0}}\right)$ als relativistisch invariant betrachtet werden kann; dies gilt nicht für das Produkt $\delta(\mathfrak{f}) \delta_{+}\left(k^{0}\right)$, da die untere Grenze des Integrals in (3) vom Koordinatensystem abhängt. Die Singularität des Ausdrucks $\delta(\mathfrak{f}) \delta_{+}\left(k^{0}\right)$ hängt jedoch nicht von der speziellen unteren Grenze im Integral (3) ab, so daß der singuläre Teil des Produktes $\delta(\mathfrak{f}) \delta_{+}\left(k^{0}\right)$ wieder als relativistisch invariant gelten kann.

b) Die Matrix $S$ kann als eine Art Transformationsfunktion betrachtet werden, die von den Impulsen vor dem Stoß zu den Impulsen nach dem Stoß transformiert. Diese Deutung legt die Annahme nahe, daß $S$ eine unitäre Matrix ist, so daß die Gleichungen

gelten.

$$
S^{\dagger} S=S S^{\dagger}:=1
$$

Der Beweis für diese wichtige Beziehung kann am einfachsten nach den Methoden der Möllerschen Arbeit ${ }^{4}$ erbracht werden, der wir uns im folgenden anschließen: Die Hamiltonsche Funktion des Systems kann in der Form $H=$ $K_{0}+V$ dargestellt werden, oder in der Diracschen Bezeichnungsweise durch

$$
\left(\mathfrak{k}^{\prime \prime}|H| \mathfrak{k}^{\prime}\right)=K_{0}^{\prime} \delta\left(\mathfrak{k}^{\prime \prime} \mathfrak{k}^{\prime}\right)+\left(\mathfrak{k}^{\prime \prime}|V| \mathfrak{k}^{\prime}\right) .
$$

Die Schrödinger-Gleichung wird dann

oder

$$
\left(K_{0}+V\right) \psi=\psi K_{0}
$$

$$
\left(\mathfrak{k}^{\prime \prime}|\psi| \mathfrak{k}^{\prime}\right)\left(K_{0}^{\prime}-K_{0}^{\prime \prime}\right)=\left(\mathfrak{k}^{\prime \prime}|V \psi| \mathfrak{k}^{\prime}\right) .
$$

Wenn man (6) auf der linken Seite einsetzt, findet man

$$
r=2 \pi i V \psi
$$

und die konjugierte Gleichung

$$
r^{\dagger}=-2 \pi i \psi^{\dagger} V \text {. }
$$

Multiplikation mit $\psi^{\dagger}$ oder $\psi$ und Addition ergibt

$$
\psi^{\dagger} r+r^{\dagger} \psi=0 \text {. }
$$

Wenn man Gleichung (15) mit dem Faktor $\delta\left(K_{0}^{\prime}-K_{0}^{\prime \prime}\right)$ multipliziert, folgt schließlich

$\left(\mathfrak{k}^{\prime \prime}\left|R+R^{\dagger}\right| \mathfrak{k}^{\prime}\right)+\delta\left(K_{0}^{\prime}-K_{0}^{\prime \prime}\right) j^{\prime}\left[\delta_{-}\left(K_{0}^{\prime \prime}-K_{0}^{\prime \prime \prime}\right)\right.$ $\left.+\delta_{+}\left(K_{0}{ }^{\prime}-K_{0}{ }^{\prime \prime \prime}\right)\right]\left(\mathfrak{k}^{\prime \prime \prime}\left|r^{*}\right| \mathfrak{k}^{\prime}\right) d \mathfrak{l}^{\prime \prime \prime}\left(\mathfrak{k}^{\prime \prime \prime}|\boldsymbol{r}| \mathfrak{k}^{\prime}\right)=0$,

oder unter Benutzung von (4):

und

$$
\begin{gathered}
R^{\dagger}+R+R^{\dagger} R=0 \\
S \dagger S=1 .
\end{gathered}
$$

Aus dieser Gleichung folgt die Gleichung mit umgekehrter Reihenfolge der Faktoren: $S S^{\dagger}=1$ nur dann, wenn man weiß, daß die reziproke Matrix $S^{-1}$ existiert. Die Existenz von $S^{-1}$ ergibt sich jedoch aus der Tatsache, daß die Wellenfunktionen $\psi$ in (6), die $\mathrm{zu}$ einem bestimmten Energiewert $K_{0}$ gehören, ein vollständiges Orthogonal-System von Wellen für diese bestimmte Energie bilden. Indem man Wellenfunktionen von der Art (6) superponiert, kann man sich eine Wellenfunktion aufbauen, die zu einer bestimmten auslaufenden ebenen Welle gehört. Wenn man mit Wellenfunktionen dieser letzteren Art die Überlegungen wiederholt, die von Gleichung (11) nach Gleichung (18) geführt haben, kann man leicht zeigen, dạ auch $S S^{\dagger}=1$ ist.

Wenn man Gl. (15) mit dem Faktor $\delta_{+}\left(K_{0}^{\prime}-K_{r}^{\prime \prime}\right)$ multipliziert und die Beziehung

$$
\left[\delta_{+}(a)+\delta_{-}(b)\right] \delta_{+}(a-b)=-\delta_{+}(a) \delta_{-}(b)
$$

benützt, so ergibt sich nach $\mathrm{M}$ ölle $\mathrm{r}^{4}$ :

$$
\psi^{\dagger} \psi=1 \text {. }
$$

Diese Beziehung bedeutet einfach, daß die Wellenfunktionen (6) im kontinuierlichen Spektrum 
richtig normiert sind. Das Produkt mit umgekehrter Reihenfolge der Faktoren: $\psi \psi^{\dagger}$ wird im allgemeinen nicht gleich 1 sein, weil die Wellenfunktionen (6) kein vollständiges OrthogonalSystem bilden, denn die Wellenfunktionen der diskreten stationären Zustände sind in der Matrix (6) nicht enthalten.

Die Gln. (17) und (18) umfassen eine Anzahl von Beziehungen zwischen beobachtbaren GröBen, die zum mindesten grundsätzlich durch Experimente geprüft werden können. Die MatrixElemente von $R$ sind unmittelbar mit den Wirkungsquerschnitten für die Streuung, Emission und Absorption von Teilchen ${ }^{6}$ verknüpft, und Gl. (17) zeigt, daß es Beziehungen gibt zwischen Termen, die linear oder quadratisch von diesen Matrix-Elementen abhängen. Eine von diesen Beziehungen ist wohlbekannt und besitzt eine einfache physikalische Deutung: Die Amplitude der Streuwelle in der Richtung des einfallenden Strahles ist verknüpft mit der Gesamtintensität der in allen Richtungen gestreuten Wellen, wobei es gleichgültig ist, welche Teilchen etwa gleichzeitig mit dieser Streuung emittiert werden. Diese Beziehung kommt dadurch zustande, daß die kohärènte Streuwelle in der Richtung des einfallenden Strahles durch Interferenz mit dem Primärstrahl den Teil der Welle wegnimmt, der in andere Richtungen gestreut wird. Gl. (17) enthält außerdem noch eine Reihe ähnlicher Beziehungen.

c) Im allgemeinen führt eine unitäre Matrix nicht zu einem Eigenwertproblem und kann nicht auf Diagonalform gebracht werden, da ihre Zeilen und Kolonnen $\mathrm{zu}$ verschiedenen Arten und verschiedenen Anzahlen von Zuständen gehören. Bei der Matrix $S$ jedoch beziehen sich Zeilen und Kolonnen auf Zustände der gleichen Art, nämlich auf die Impulse der einfallenden oder der auslaufenden Teilchen. Deshalb kann die Matrix $S$ auf Diagonalform gebracht werden.

Die Aufgabe, diese Transformation zu finden, kann auf das folgende mathematische Problem reduziert werden: Die Wellenfunktionen der Art (6), die zu einer bestimmten Energie gehören, müssen in einer solchen Weise superponiert werden, daß für die resultierende Wellenfunktion die einfallende Welle die gleiche Form hat wie die auslaufende und sich von ihr nur durch einen

\footnotetext{
6 Die Formeln 37 a) und b) von I für die Wirkungsquerschnitte sind durch einen Rechenfehler entstellt, der von $\mathrm{M}$ öll ${\mathrm{e}{ }^{4}}^{4}$ verbessert worden ist.
}

Phasenfaktor unterscheidet. Dieser Phasenfaktor ist dann der Eigenwert von $S$ und kann dargestellt werden durch:

$$
S^{\prime}=\mathrm{e}^{i \eta^{\prime}}
$$

Tatsächlich ist dieses mathematische Verfahren in einem Spezialfall oft angewendet worden, nämlich für die Streuung von Teilchen in einem zentralen Kraftfeld. Hier muß man einfach von, den ebenen Wellen zu Kugelwellen übergehen; dann haben die einfallenden und auslaufenden Wellen die gleiche Form, und die Phasendifferenzen zwischen einfallenden und auslaufenden Wellen für die einzelnen Kugelfunktionen bestimmen den Wirkungsquerschnitt für Streuung.

Wenn man von den Eigenwerten von $S$ auf die ursprüngliche Matrix zurücktransformiert, so erhält man aus den Eigenwerten $\eta^{\prime}$ eine hermitesche Matrix $\eta$, die mit $S$ durch die Beziehung

$$
S=\mathrm{e}^{i} \|
$$

verbunden ist. Diese hermitesche Matrix $\eta$ soll als Phasenmatrix des Systems bezeichnet werden.

Gl. (21) kann benutzt werden, um die relativistischen Eigenschaften der Matrix $S$ zu studiercn. Die Phase einer Welle ist relativistisch invariant; das gleiche gilt daher auch für die Eigenwerte von $\eta$ und $S$. Eine Matrix mit Eigenwerten, die relativistisch invariant sind, kann eine invariante Matrix genannt werden. Dies bedeutet jedoch nicht, daß die einzelnen Matrix-Elemente von $S$ oder $\eta$ invariante Funktionen ihrer Argumente sind. Die Matrix-Elemente, die zu Übergängen zwischen zwei diskreten stationären Zuständen gehören, würden natürlich nicht vom Bezugssystem abhängen. Aber im kontinuierlichen Spektrum sind die Matrix-Elemente Funktionen der Impulse $\mathfrak{f}$, die den Zustand charakterisieren. Der Differentialausdruck $d \mathfrak{f}_{x} d \mathfrak{f}_{y} d \mathfrak{f}_{z}$ ist gegenüber einer Lorentz-Transformation nicht invariant, während der Ausdruck

$$
d \mathfrak{k}_{x} d \mathfrak{k}_{y} d \mathfrak{k}_{z} / k^{0}
$$

eine Invariante darstellt. Deshalb müssen die Matrix-Elemente einer invarianten Matrix im kontinuierlichen Spektrum die Form

$$
\left(\mathfrak{l}^{\prime \prime}|A| \mathfrak{k}^{\prime}\right)=\frac{\text { Invariante Funktion der } \mathfrak{k}_{i}^{\prime} \text { u. } \hat{\mathfrak{k}}_{i}^{\prime \prime}}{\sqrt{\prod_{l} k_{i}{ }^{0 \prime} \cdot \prod_{l} k_{l}{ }^{0 \prime \prime}}}
$$


haben. Denn wenn (23) gilt, so sorgt der Normierungsfaktor im Nenner dafür, daß bei der Multiplikation von zwei solchen Matrizen das Differential $\prod_{i} d \mathfrak{k}_{i x}{ }^{\prime} d \mathfrak{k}_{i y}{ }^{\prime} d \mathfrak{k}_{i z}{ }^{\prime}$ vervollständigt wird $\mathrm{zu}$ dem invarianten Ausdruck

$$
\prod_{i} \frac{d \mathfrak{k}_{i x}^{\prime} d \mathfrak{k}_{i y}^{\prime} d \mathfrak{k}_{i z}^{\prime}}{k_{i}^{0 \prime}}
$$

d) Bevor behauptet werden kann, daß die unitäre Matrix $S$ oder die hermitesche Matrix $\eta$ alle notwendigen Informationen über die beobachtbaren Größen enthalten, müssen noch zwei weitere Fragen untersucht werden. Erstens scheint $S$ oder $\eta$ keine Auskunft zu geben über die diskreten stationären Zustände des Systems. Zweitens gibt es, wenn das System aus vielen Teilchen besteht, nicht nur eine auslaufende und eine einfallende Welle, sondern es gibt auch Wellen, bei denen einige der Teilchen sich nach innen, andere nach außen bewegen. Daher entsteht die Frage, ob das asymptotische Verhalten dieser Wellen durch 5 beschrieben wird.

Bei der Untersuchung der ersten Frage wollen wir der Einfachheit halber annehmen, daß es sich um ein einzelnes Teilchen in einem zentralen Kraftfeld handelt. Dann können $S$ und $\eta$ auf Diagonalform gebracht werden, indem man Wellenfunktionen benützt, die Produkte von Kugelfunktionen und Funktionen des absoluten Wertes des Impulses $k$ sind. In. großen Abständen vom Streuzentrum hat die einfallende Welle die Form $e^{-i h r} / r$, die auslaufende lautet $e^{i k r} / r$. Die Eigenwerte $S^{\prime}$ von $S$ bestimmen den Faktor von $e^{i k r} / r$, wenn die einfallende Welle ohne irgendeinen Phasenfaktor angenommen wird. Der Eigenwert $S^{\prime}$ ist eine Funktion von $k$ und damit von der Energie des Systems, und man kann zeigen, wie von

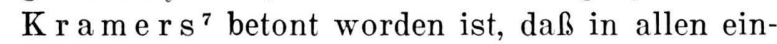
fachen Fällen $S^{\prime}$ eine analytische Funktion von $k$ ist. Wenn dies richtig ist, so wird $S^{\prime}$ auch seine Bedeutung behalten, wenn man zu negativen Energiewerten, d. h. zu komplexen Impulswerten von $k$ übergeht. Wir wollen annehmen, daß in diesem Gebiet $k=+i|k|$ ist. Dann geht die einfallende Welle über in $e^{+|k| r / r}$, die auslaufende wird

7 H. A. K r a m e r s hat die Verknüpfung von $S$ mit den diskreten stationären Zuständen des Systems gefunden und hat sie mir freundlicherweise in einer Diskussion in Leiden 1942 auseinandergesetzt. $e^{-|k| r / r}$. Der Eigenwert $S^{\prime}$ gibt das Verhältnis des Teiles $e^{-|k| r / r}$ relativ zu dem Teil $e^{+|k| r / r}$ für die gegebene Energie. Nun enthält in einem diskreten stationären Zustand die Lösung der Wellengleichung nur den Teil $e^{-|k| r / r}$. Daher muß $S^{\prime}$ unendlich werden für die Energie der diskreten stationären Zustände des Systems; in anderen Worten: die diskreten stationären $\mathrm{Zu}$ stände sind gegeben durch die Pole von $S^{\prime}$ auf der imaginären Achse im komplexen $k$-Raum. Eine nähere Untersuchung zeigt, daß $S^{\prime}$ im allgemeinen einen Faktor $\frac{k+i\left|k_{n}\right|}{k-i\left|k_{n}\right|}$ enthalten wird, wenn $k=i\left|k_{n}\right|$ ein stationärer Zustand des Systems ist. Die Eigenwerte $\eta^{\prime}$ von $\eta$ divergieren logarithmisch an den Punkten $k= \pm i\left|k_{n}\right| \cdot \eta^{\prime}(k)$ ist immer eine ungerade Funktion von $k: \eta^{\prime}(-k)=-\eta^{\prime}(k)$.

Diese Betrachtungen zeigen, daß man die stationären Zustände des Systems stets aus der Matrix $S$ ableiten kann, indem man die Pole und Nullstellen von $S^{\prime}$ als Funktion von $k$ aufsucht. Zum Beispiel kann man dieses Verfahren beim C o u l o m b schen Kraftfeld anwenden. In diesem Falle enthält die Matrix $S$ (oder richtiger: ihre Eigenwerte $S^{\prime}$ ) den Faktor ${ }^{8}$

$$
\frac{\Gamma\left(1+l-i \frac{x}{k}\right)}{\Gamma\left(1+l+i \frac{x}{k}\right)},
$$

wobei $l$ die azimutale Quantenzahl bedeutet und $\varkappa=\frac{Z e^{2} m}{\hbar^{2}}(Z e$ ist die Kernladung, $e$ und $m$ bedeuten Ladung und Masse des Elektrons.) Da die Funktiōn $\Gamma(x)$ Pole für $x=0$ und für alle negativen ganzzahligen Werte von $x$ besitzt, sind die Pole von $S^{\prime}$ gegeben durch:

$$
k=\frac{i x}{n},
$$

wobei $n$ eine ganze $\mathrm{Zahl} \geqslant l+1$ ist.

Da die Energie in gewöhnlichen Einheiten durch $E=\frac{\hbar^{2}}{2 m} k^{2}$ gegeben ist, findet man ohne weiteres die B almer-Formel:

8 Vergl. H. B e th e, Handbuch d. Physik, Bd. 24, 1, S. 291 [1933]. 


$$
E=-\frac{Z^{2} e^{4} m}{2 n^{2} \hbar^{2}}
$$

Außer den Energiewerten der diskreten stationären Zustände gibt die Matrix $S$ auch den Normierungsfaktor für die Wellenfunktionen in diesen Zuständen. Dies kann in folgender Weise gezeigt werden: Im kontinuierlichen Spektrum ist die Wellenfunktion asymptotisch gegeben durch:

$$
\psi_{r \rightarrow \infty}=\frac{\sin \left[k r+\frac{1}{2} \eta^{\prime}(k)\right]}{r \pi \sqrt{\zeta}} .
$$

Man kann leicht zeigen, daß diese Funktion richtig normiert ist, unabhängig von den speziellen Werten der Phase $\eta^{\prime}(k)$ und unabhängig vom Verhalten der Wellenfunktion bei kleinen Werten von $r$.

$$
\int_{0}^{\infty} 4 \pi r^{2} d r \psi_{k}(r) \psi_{k^{\prime}}(r)=\delta\left(k-k^{\prime}\right) .
$$

Die Wellenfunktion eines diskreten stationären Zustandes muß sich bei großen Abständen wie

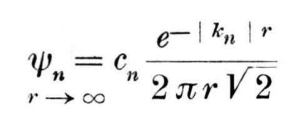

verhalten. Hier würde jedoch die Annahme $c_{n}=1$ sicher nicht die richtigeNormierung geben, da $c_{n}{ }^{2}$ die Dimension einer reziproken Länge haben muß. Um die Werte von $c$ zu bestimmen, betrachten wir die Vollständigkeitsrelation

$$
\begin{array}{r}
\int^{\infty} d k \psi_{k}^{*}(r) \psi_{k}\left(r^{\prime}\right)+\sum_{n} \psi_{n}^{*}(r) \psi_{n}\left(r^{\prime}\right) \\
=\frac{1}{4 \pi r^{2}} \delta\left(r-r^{\prime}\right) .
\end{array}
$$

Diese Beziehung muß auch für große Werte von $r$ und $\boldsymbol{r}^{\prime}$ gelten. Wenn man (24) und (26) in Gl. (27) einsetzt, so ergibt sich:

$$
\begin{aligned}
& \int_{0}^{\infty} \frac{d k}{2 \pi^{2} r^{2}} \sin \left[k r+\frac{1}{2} \eta^{\prime}(k)\right] \sin \left[k r^{\prime}+\frac{1}{2} \eta^{\prime}(k)\right] \\
& +\frac{1}{8 \pi^{2} r^{2}} \sum_{n}\left|c_{n}{ }^{2}\right| e^{-\left|k_{n}\right|\left(r+r^{\prime}\right)}=\frac{1}{4 \pi r^{2}} \delta\left(r-r^{\prime}\right) .
\end{aligned}
$$

Unter Benutzung der Beziehung

$$
\eta^{\prime}(-k)=-\eta^{\prime}(k)
$$

geht (28) über in

$$
\int_{-\infty}^{+\infty} d k e^{i k\left(r+r^{\prime}\right)+i \eta^{\prime}(k)}=\sum_{n}\left|c_{n}{ }^{2}\right| e^{-\left|k_{n}\right|\left(r+r^{\prime}\right)}
$$

Nun hat der Faktor $e^{i} \eta^{\prime}=S^{\prime}(k)$ einen Pol an jedem Punkt $k=i\left|k_{n}\right|$ auf der imaginären $k$-Achse. Wenn man den Integrationsweg in Richtung auf positiv imaginäre Werte von $k$ verschiebt, so reduziert er sich schließlich auf Kreise um die Pole $i\rfloor k_{n}$, , und das Residuum bei jedem einzelnen Pol entspricht einem Glied der Summe auf der rechten Seite. So erhält man schließlich ${ }^{9}$ :

$$
\left|c_{n}^{2}\right|=\oint_{i \mid k_{n} !} d k S^{\prime}(k)
$$

In dieser Weise wird der absolute Wert des Normierungsfaktors $c_{n}$ durch die S-Matrix bestimmt. Die Phase von $c_{n}$ bleibt unbestimmt, aber die Phase einer Wellenfunktion in einem diskreten stationären Zustande gehört nicht $\mathrm{zu}$ den beobachtbaren Größen.

Die Beziehung (31) gestattet, die diskreten Matrix-Elemente einer gegebenen Matrix als analytische Forlsetzung der Elemente im kontinuierlichen Spektrum zu bestimmen. Tatsächlich sollte man für jede Matrix $A$ die Beziehung

$$
\left(k^{\prime}\left|A^{*}\right| n\right)\left(k^{\prime \prime}|A| n\right)=-\oint_{i\left|k_{n}\right|} d k\left(k^{\prime}\left|A^{*}\right| k\right)\left(k^{\prime \prime}|A| k\right)
$$

erwarten, es sei denn, daß die Matrix $A$ selbst Singularitäten an den Punkten $k=i\left|k_{n}\right|$ einführt. Gl. (32) kann durch die aus (27) folgende Beziehung

$$
\psi_{n}^{*}(r) \psi_{n}\left(r^{\prime}\right)=-\oint_{i\left|k_{n}\right|} d k \psi_{k}^{*}(r) \psi_{k}\left(r^{\prime}\right)
$$

begründet werden. In diesen Gln. (32) und (33) bedeutet der Stern, daß der konjugiert komplexe Wert zu nehmen ist im Fall von reellen Werten von $k$. Für komplexe Werte von $\mathrm{k}$ auf der rechten Seite soll die analytische Fortsetzung der Werte für reelle $k$ genommen werden. Gl. (32) gilt für gewöhnliche Matrizen, die z.B. durch gewisse Funktionen der Teilchen-Koordinaten, wie potentielle und kinetische Energie usw., definiert sind,

${ }^{9}$ Im wesentlichen die gleiche Beziehung ist auch von $\mathrm{Ch}$. M öller gefunden worden, der sie mir freundlicherweise in einem Brief im Sommer 1944 mitgeteilt hat. 
aber sie gilt vielleicht nicht für Matrizen wie $S$ und $\eta$, deren Eigenwerte singulär sind an den Punkten $k=i\left|k_{n}\right|$. Deshalb erfordert die Frage nach der Gültigkeit von (32) eine sorgfältige Untersuchung in jedem einzelnen Fall.

e) Wenn das System aus mehreren Teilchen besteht, so bestimmt die Matrix $S$ das asymptotische Verhalten der Wellen nicht in derselben einfachen Weise wie für ein Teilchen. Um diesen Fall zu studieren, betrachten wir ein System von zwei Teilchen ohne Wechselwirkung, die an einem zentralen Kraftfeld gestreut werden. Wenn die Teilchen allein wären, würden ihre Wellenfunktionen lauten

$$
\begin{aligned}
\psi_{1} & =\delta\left(\mathfrak{k}_{1}{ }^{\prime \prime} \mathfrak{k}_{1}{ }^{\prime}\right)+\delta_{+}\left(k_{1}{ }^{0 \prime}-k_{1}{ }^{0 \prime \prime}\right)\left(\mathfrak{k}_{1}{ }^{\prime \prime}\left|r_{1}\right| \mathfrak{k}_{1}{ }^{\prime}\right), \\
\psi_{2} & =\delta\left(\mathfrak{k}_{2}{ }^{\prime \prime} \mathfrak{k}_{2}{ }^{\prime}\right)+\delta_{+}\left(k_{2}{ }^{0 \prime}-k_{2}{ }^{0 \prime \prime}\right)\left(\mathfrak{k}_{2}{ }^{\prime \prime}\left|r_{2}\right| \mathfrak{k}_{2}{ }^{\prime}\right) .
\end{aligned}
$$

Die Gesamtwellenfunktion ist das Produkt von $\psi_{1}$ und $\psi_{2}$, da es keine Wechselwirkung zwischen den Teilchen geben soll, und dieses Produkt kann in der folgenden Weise umgeschrieben werden

$$
\begin{aligned}
& \psi=\psi_{1} \psi_{2}=\delta\left(\mathfrak{k}_{1}{ }^{\prime \prime} \mathfrak{k}_{1}{ }^{\prime}\right) \delta\left(\mathfrak{k}_{2}{ }^{\prime \prime} \mathfrak{k}_{2}{ }^{\prime}\right)+\delta_{+}\left(K_{0}{ }^{\prime}-K_{0}{ }^{\prime \prime}\right) \\
& \left\{\delta\left(\mathfrak{k}_{2}{ }^{\prime \prime} \mathfrak{k}_{2}{ }^{\prime}\right)\left(\mathfrak{k}_{1}{ }^{\prime \prime}\left|r_{1}\right| \mathfrak{k}_{1}{ }^{\prime}\right)+\delta\left(\mathfrak{k}_{1}{ }^{\prime \prime} \mathfrak{k}_{1}{ }^{\prime}\right)\left(\mathfrak{k}_{2}{ }^{\prime \prime}\left|\boldsymbol{r}_{2}\right| \mathfrak{k}_{2}{ }^{\prime}\right)\right. \\
& +\left[\delta_{+}\left({k_{1}}^{0 \prime}-k_{1}{ }^{0 \prime \prime}\right)\right. \\
& \left.\left.+\delta_{+}\left(k_{2}{ }^{0 \prime}-k_{2}{ }^{0 \prime \prime}\right)\right]\left(\mathfrak{k}_{1}{ }^{\prime \prime}\left|r_{1}\right| \mathfrak{k}_{1}{ }^{\prime}\right)\left(\mathfrak{k}_{2}{ }^{\prime \prime}\left|r_{2}\right| \mathfrak{k}_{2}{ }^{\prime}\right)\right\} .
\end{aligned}
$$

Der Klammerausdruck auf der rechten Seite, der mit $\delta_{+}\left(K_{0}{ }^{\prime}-K_{0}{ }^{\prime \prime}\right)$ multipliziert ist, kann wie gewöhnlich als $\left(\mathfrak{k}_{1}{ }^{\prime \prime} \mathfrak{k}_{2}{ }^{\prime \prime}|r| \mathfrak{k}_{1}{ }^{\prime} \mathfrak{k}_{2}{ }^{\prime}\right)$ bezeichnet werden; dann zeigt Gl. (35), daß die Matrix $r$ singulär ist an den Stellen $\mathfrak{f}_{1}^{\prime}=\mathfrak{f}_{1}{ }^{\prime \prime}$ und $\mathfrak{f}_{2}{ }^{\prime}=\mathfrak{f}_{2}{ }^{\prime \prime}$, und daß die Verknüpfung von $r$ mit $r_{1}$ und $r_{2}$ ziemlich verwikkelt ist. Das asymptotische Verhalten der Wellen ist durch die Art der Singularität bestimmt; es ist daher offenbar nicht ausreichend, den Wert von $\left(\mathfrak{k}_{1}{ }^{\prime \prime} \mathfrak{k}_{2}{ }^{\prime \prime}|r| \mathfrak{k}_{1}{ }^{\prime} \mathfrak{k}_{2}{ }^{\prime \prime}\right)$ für $K_{0}{ }^{\prime}=K_{0}{ }^{\prime \prime}$ zu kennen, sondern es ist notwendig, $r$ in einer infinitesimalen Umgebung dieses Punktes zu kennen, um das asymptotische Verhalten der Wellen $\mathrm{zu}$ bestimmen. Trotzdem ergibt sich eine einfache Verknüpfung zwischen dem Problem der zwei Teilchen ohne Wechselwirkung und den Problemen der einzelnen Teilchen, wenn man von der Matrix $r$ zu den Matrizen $R$ oder $S$ übergeht. Wenn man nämlich den Klammerausdruck auf der rechten Seite von (35) mit $\delta\left(K_{0}{ }^{\prime}-K_{0}{ }^{\prime \prime}\right)$ multipliziert, erhält man sofort

$$
\begin{aligned}
& R=R_{1}+R_{2}+R_{1} R_{2} \\
\text { oder } & S=S_{1} S_{2}, \\
\text { und da } & S=e^{i \eta}: \eta=\eta_{1}+\eta_{2} .
\end{aligned}
$$

Daher ist die S-Matrix eines Systems von zwei Teilchen ohne Wechselwirkung gleich dem Produkt der $S$-Matrizen für die einzelnen Teilchen. Die $\eta$-Matrix ist gleich der Summe der einzelnen $\eta$-Matrizen. Aber die $S$-Matrix bestimmt das asymptotische Verhalten der Wellen nicht unmittelbar; sie bestimmt es nur in Verbindung mit $S_{1}$ und $S_{2}$, da die singulären Teile der Klammer in (35) aus $S_{1}$ und $S_{2}$ abgeleitet werden können. Die letztere Behauptung gilt auch, wenn die beiden Teilchen miteinander in Wechselwirkung stehen, da ihre Wechselwirkung die singulären Teile des Klammerausdrucks nicht verändert.

Eine Wechselwirkung zwischen den beiden Teilchen kann dann verhältnismäßig leicht mathematisch behandelt werden, wenn man annimmt, daß die Wechselwirkung sehr klein und durch eine kleine potentielle Energie $v$ gegeben ist. Wir definieren dann eine Matrix $V$ durch

$\left(\mathfrak{f}_{1}^{\prime \prime} \mathfrak{k}_{2}^{\prime \prime}|V| \mathfrak{k}_{1}^{\prime} \mathfrak{k}_{2}{ }^{\prime}\right)=\delta\left(K_{0}^{\prime}-K_{0}{ }^{\prime \prime}\right)\left(\mathfrak{k}_{1}^{\prime \prime} \mathfrak{k}_{2}^{\prime \prime}|\boldsymbol{v}| \mathfrak{k}_{1}{ }^{\prime} \mathfrak{k}_{2}{ }^{\prime}\right)$.

Eine einfache Rechnung zeigt (Teil IV der unter ${ }^{3}$ zitierten Arbeiten), daß die Matrix $S$ in dieser Näherung durch

$$
\dot{S}=S_{1} S_{2}(1-2 \pi i V)=S_{1} S_{2} \mathrm{e}^{-\pi i v}
$$

gegeben ist.

Dies bedeutet jedoch nicht, daß $\eta$ durch $\eta_{1}+\eta_{2}-2 \pi i V$ gegeben wäre, da $V$ im allgemeinen nicht mit $S_{1}, S_{2}$ oder $\eta_{1}$ und $\eta_{2}$ vertauschbar ist.

Gl. (40) hat zur Folge, daß innerhalb der benutzten Näherung die Gl. (32) auch für die Matrix $S$ gilt:

$$
\begin{aligned}
& \left(k_{1}{ }^{\prime}, k_{2}{ }^{\prime}\left|S^{*}\right| n, k_{2}\right)\left(k_{1}{ }^{\prime \prime} k_{2}{ }^{\prime \prime}|S| n, k_{2}\right) \\
& =-\oint_{i \mid k_{n}} d k_{1}\left(k_{1}{ }^{\prime} k_{2}{ }^{\prime}\left|S^{*}\right| k_{1} k_{2}\right)\left(k_{1}{ }^{\prime \prime} k_{2}{ }^{\prime \prime}|S| k_{1} k_{2}\right),
\end{aligned}
$$

da sie für $V$ richtig ist. Aber Gl. (32) gilt im allgemeinen nicht für die Matrix $\eta$, die an den singulären Punkten $k_{n}$ der diskreten stationären $\mathrm{Zu}$ stände logarithmisch divergiert.

Bei der Ableitung von (41) war angenommen worden, daß die Wechselwirkung zwischen den Teilchen klein sei. Das einfache Ergebnis legt 
jedoch die Annahme nahe, daß (41) für jede beliebige Wechselwirkung gilt. Ein allgemeiner Beweis für (41) würde eine sehr sorgfältige Untersuchung des Verhaltens von $S$ in der Nähe der singulären Punkte voraussetzen und soll hier nicht versucht werden.

Gl. (41) zeigt, daß die Matrix $S$ nicht nur die Lage der diskreten stationären Zustände $\mathrm{zu}$ bestimmen gestattet, sondern auch die Wahrscheinlichkeiten für die Anregung solcher Zustände oder für die Streuung von Teilchen an diesen Zuständen. Man kann daraus schließen, daß die Matrizen $S$ oder $\eta$ tatsächlich all die Informationen umfassen, die für die Deutung irgendeines am System vorgenommenen Experimentes notwendig sind.

\section{Bestimmung der Matrix $S$}

a) In Anbetracht der grundsätzlichen Wichtigkeit der Matrix $S$ oder $\eta$ entsteht die Frage, wie man für ein gegebenes System die richtige $S$-Matrix bestimmen kann. Bisher hat man die Bestimmung der $S$-Matrix für das gegebene System (z. B. für Elektronen und ihr $\mathrm{Maxwellsches} \mathrm{Feld)}$ in der folgenden Weise versucht. Zunächst definiert man eine $\mathrm{Hamilt}$ on sche Funktion, die man aus Korrespondenzbetrachtungen oder durch Invarianzbedingungen nach dem Grundsatz möglichster Einfachheit zu gewinnen sucht, und dann leitet man die $S$-Matrix ab, indem man die zur Hamilton-Funktion gehörige Wellengleichung löst. Diesem Verfahren stehen jedoch zwei große Schwierigkeiten im Wege: Die HamiltonFunktionen für Wellenfelder führen, soweit man sie bisher studiert hat, zu Divergenzen, und die Verknüpfung der verschiedenen Wellenfelder bringt von selbst Verallgemeinerungen der Wellengleichungen mit sich, die Zweifel hinsichtlich der Annahme aufkommen lassen, daß die Hamilton sche Funktion, mit der man beginnt, eine einfache Form haben soll. Diese beiden Schwierigkeiten könnten sich möglicherweise kompensieren. Es könnte sein, daß es Hamilton-Funktionen der allgemeineren Art gibt, die zu vernünftigen Resultaten führen.

Um diese Möglichkeit näher zu verfolgen, werden wir die Verallgemeinerungen betrachten, die

10 M. B o rn , Proc. Roy. Soc. [London] (A) 143, 410 [1933]; M. B or n u. L. In f eld, Proc. Roy. Soc. [London] (A) 144, 425 [1934]; 147, 522 [1934]; 150, 141 [1935]. sich aus der Wechselwirkung verschiedener Wellenfelder und aus der Diracschen Löchertheorie ergeben. Die Wechselwirkung zwischen verschiedenen Feldern führt zu nichtlinearen Gliedern in der Wellengleichung. Deshalb kann angenommen werden, daß die richtigen Wellengleichungen nichtlineare Glieder enthalten, wie es z. B. für die Quantenelektrodynamik schon früher von B o r n und anderen ${ }^{10}$ vorgeschlagen worden ist. Die D i racsche Löchertheorie andererseits setzt an die Stelle der Maxwellschen Gleichungen IntegroDifferentialgleichungen, wie von $\mathrm{R}$. Serber ${ }^{11}$ und E. A. U e hling ${ }^{12}$ gezeigt worden ist. Deshalb liegt die Annahme nahe, daß die richtige Wellengleichung eine Integro-Differentialgleichung sein kann. Lineare Wellengleichungen dieser Art sind von E. C. G. St ü ckel be r g ${ }^{13}$, $\mathrm{F}$. B o p p ${ }^{14}$ und anderen erörtert worden.

Wenn man diese beiden Verallgemeinerungen als die wichtigsten betrachtet und der Einfachheit halber annimmt, daß es nur eine skalare Wellenfunktion geben soll, so erhält man eine Wellengleichung von der Form

$$
\begin{aligned}
& \quad \int d x^{\prime} A\left(x-x^{\prime}\right) \varphi\left(x^{\prime}\right) \\
& +\int d x^{\prime} d x^{\prime \prime} B\left(x-x^{\prime}, x-x^{\prime \prime}\right) \varphi\left(x^{\prime}\right) \varphi\left(x^{\prime \prime}\right) \\
& +\ldots=0
\end{aligned}
$$

oder, unter Benutzung der F o u ri i r - Transformation:

$$
\varphi(x)=\int e^{i k_{\mu} x \mu} \varphi(k) d k
$$

eine Wellengleichung im Impulsraum von der Form

$$
\begin{array}{r}
A(k) \varphi(k)+\int d k^{\prime} B\left(k, k^{\prime}\right) \varphi\left(k^{\prime}\right) \varphi\left(k-k^{\prime}\right) \\
+\ldots=0 .
\end{array}
$$

Hier bezeichnen $x$ und $k$ die Vierer-Vektoren $x_{1} \ldots x_{4}$ und $k_{1} \ldots k_{4} ; A$ und $B$ sind relativistisch invariante Funktionen ihrer Argumente. Gl. (43) führt $\mathrm{zu}$ einem Energieimpuls-Tensor, der, wie gewöhnlich, nicht eindeutig festgelegt ist. Eine mögliche Form dieses Tensors ist (wieder im Impulsraum) :

\footnotetext{
11 Physic. Rev. 48, 49 [1935̃].

12 Physic. Rev. 48, 55 [1935].

13 Helv. physica Acta 17, 3 [1944].

14 Ann. Physik 38, 549 [1940]; 42, 572 [1943]; Z. Naturforschg. 1, 53 [1946].
} 


$$
\begin{aligned}
T_{\mu \nu}(k) & =\int d k^{\prime} A\left(k^{\prime}\right) \varphi\left(k^{\prime}\right) \varphi\left(k-k^{\prime}\right) \frac{k_{\mu}{ }^{\prime} k_{\nu}}{k_{\lambda}{ }^{\prime} k_{\lambda}} \\
& +\int d k^{\prime} d k^{\prime \prime} B\left(k^{\prime} k^{\prime \prime}\right) \varphi\left(k^{\prime \prime}\right) \varphi\left(k^{\prime}-k^{\prime \prime}\right) \varphi\left(k-k^{\prime}\right)\left\{\frac{k_{\mu}{ }^{\prime \prime} k_{\nu}{ }^{\prime \prime}}{k_{\lambda}^{\prime \prime} k_{\lambda}}+\frac{\left(k_{\mu}{ }^{\prime}-k_{\mu}{ }^{\prime \prime}\right)\left(k_{\nu}{ }^{\prime}-k_{\nu}{ }^{\prime \prime}\right)}{\left(k_{\lambda}^{\prime}-k_{\lambda}{ }^{\prime \prime}\right) k_{\lambda}}\right\} .
\end{aligned}
$$

Man erkennt leicht, daß

$$
\sum_{\nu} T_{\mu \nu}(k) k_{v}=0
$$

wie durch die Erhaltungssätze gefordert wird. Obwohl $T_{\mu \nu}$ nicht eindeutig bestimmt ist, sind die Ausdrücke für die totale Energie und den totalen Impuls wahrscheinlich eindeutig bestimmt, wobei Gl. (44) im allgemeinen bei der Berechnung dieser Größen einen komplizierten Grenzübergang nötig macht, der sorgfältig untersucht werden muß. Für den Vierer-Vektor $J_{\mu}$ der Gesamtenergie und des Gesamtimpulses findet man:

$$
\begin{aligned}
J_{\mu} & =i \int T_{\mu_{4}}(x) d x_{1} d x_{2} d x_{3} \\
& =i \int e^{i k_{4} x_{4}} T_{\mu_{4}}\left(\begin{array}{llll}
0 & 0 & 0 & k_{4}
\end{array}\right) d k_{4} .
\end{aligned}
$$

Da $T_{\mu_{4}}\left(000 k_{4}\right)$ nach (45). nur für $k_{4}=0$ von Null verschieden ist, erhält man

$$
J_{\mu}=i \int_{-}^{+} T_{\mu_{4}}\left(0000 k_{4}\right) d k_{4},
$$

wobei das Integral von irgendeinem negativen $\mathrm{zu}$ irgendeinem positiven Wert von $k_{4}$ über den Punkt $k_{4}=0$ geführt werden muß.

b) Die Quantisierung einer derartigen Theorie kann nicht in der gewöhnlichen Weise durchgeführt werden, da die Wellengleichung keine Differential-, sondern eine Integro-Differentialglei- chung ist. Man kann statt dessen auch sagen, daß die Wellengleichung unendlich hohe Ableitungen der Wellenfunktion nach den Raum- und Zeitkoordinaten enthält. In diesem Fall kann man die gewöhnlichen Vertauschungsregeln für die Wellenfunktion und ihre Ableitungen offenbar nicht verwenden. Aber es stellt sich heraus, daß die Vertauschungsregeln zwischen der Wellenfunktion und dem Energieimpulsvektor verwendet werden können und eben genügen, um die Quantisierung des Problems durchzuführen ${ }^{15}$. Diese Vertauschungsregeln fordern, daß

$$
\begin{aligned}
J_{\mu} \varphi(x)-\varphi(x) J_{\mu} & =i \hbar \frac{\partial \varphi}{\partial x_{\mu}} \\
\text { oder } \quad & J_{\mu} \varphi(k)-\varphi(k) J_{\mu}=-k_{\mu} \varphi(k) .
\end{aligned}
$$

Wenn man eine Matrixdarstellung benutzt, in der die $J$ auf Diagonalform gebracht sind, findet man

$$
\left(J_{\mu}{ }^{\prime}-J_{\mu}{ }^{\prime \prime}+k_{\mu}\right)\left(J^{\prime}|\varphi(k)| J^{\prime \prime}\right)=0 ;
$$

daraus folgt weiter

$$
\begin{aligned}
& \left(J^{\prime}|\varphi(k)| J^{\prime \prime}\right)=\delta\left(J^{\prime}-J^{\prime \prime}+k\right)\left(J^{\prime}|\varphi| J^{\prime \prime}\right), \\
& \left(J^{\prime}|\varphi(x)| J^{\prime \prime}\right)=\left(J^{\prime}|\varphi| J^{\prime \prime}\right) e^{i\left(J_{\mu}^{\prime \prime}-J^{\prime}\right) x \mu} .
\end{aligned}
$$

In diesen Formeln bedeutet

$$
\delta(k)=\delta\left(k_{1}\right) \delta\left(k_{2}\right) \delta\left(k_{3}\right) \delta\left(k_{4}\right) .
$$

Die Wellengleichung geht dann über in eine Gleichung für die Matrix $\left(J^{\prime}|\varphi| J^{\prime \prime}\right)$ :

$$
A\left(J^{\prime}-J^{\prime \prime}\right)\left(J^{\prime}|\varphi| J^{\prime \prime}\right)+\int d J^{\prime \prime \prime} B\left(J^{\prime \prime}-J^{\prime}, J^{\prime \prime \prime}-J^{\prime}\right)\left(J^{\prime}|\varphi| J^{\prime \prime \prime}\right)\left(J^{\prime \prime \prime}|\varphi| J^{\prime \prime}\right)+\ldots=0 .
$$

Der Energieimpuls-Tensor wird:

$$
\begin{aligned}
& \left(J^{\prime}\left|T_{\mu \nu}\right| J^{\prime \prime}\right)=\int d J^{\prime \prime \prime} A\left(J^{\prime \prime \prime}-J^{\prime}\right)\left(J^{\prime}|\varphi| J^{\prime \prime \prime}\right)\left(J^{\prime \prime \prime}|\varphi| J^{\prime \prime}\right) \frac{\left(J_{\mu}^{\prime \prime \prime}-J_{\mu}{ }^{\prime}\right)\left(J_{\nu}{ }^{\prime \prime \prime}-J_{\nu}^{\prime}\right)}{\left(J_{\lambda}^{\prime \prime \prime}-J_{\lambda}\right)\left(J_{\lambda}^{\prime \prime}-J_{\lambda}{ }^{\prime}\right)} \\
& +\int d \cdot J^{\prime \prime \prime} d J^{\prime \prime \prime \prime} B\left(J^{\prime \prime \prime \prime}-J^{\prime}, J^{\prime \prime \prime}-J^{\prime}\right)\left(J^{\prime}|\varphi| J^{\prime \prime \prime}\right)\left(J^{\prime \prime \prime}|\varphi| J^{\prime \prime \prime \prime}\right)\left(J^{\prime \prime \prime \prime}|\varphi| J^{\prime \prime}\right) \text {. } \\
& \cdot\left\{\frac{\left(J_{\mu}{ }^{\prime \prime \prime}-J_{\mu}{ }^{\prime}\right)\left(J_{\nu}{ }^{\prime \prime \prime}-J_{\nu}{ }^{\prime}\right)}{\left(J_{\lambda}{ }^{\prime \prime \prime}-J_{\lambda}{ }^{\prime}\right)\left(J_{\lambda}{ }^{\prime \prime}-J_{\lambda}{ }^{\prime}\right)}+\frac{\left(J_{\mu}{ }^{\prime \prime \prime \prime}-J_{\mu}{ }^{\prime \prime \prime}\right)\left(J_{\nu}{ }^{\prime \prime \prime \prime}-J_{\nu}{ }^{\prime \prime \prime}\right)}{\left(J_{\lambda}{ }^{\prime \prime \prime \prime}-J_{\lambda}{ }^{\prime \prime \prime}\right)\left(J_{\lambda}{ }^{\prime \prime}-J_{\lambda}{ }^{\prime}\right)}\right\}+\ldots
\end{aligned}
$$

und für den Vierervektor erhält man:

$$
\left(J^{\prime}\left|J_{\mu}\right| J^{\prime \prime}\right)=J_{\mu}{ }^{\prime} \delta\left(J^{\prime}-J^{\prime \prime}\right)=\delta\left(J_{1}{ }^{\prime}-J_{1}{ }^{\prime \prime}\right) \delta\left(J_{2}{ }^{\prime}-J_{2}{ }^{\prime \prime}\right) \delta\left(J_{3}{ }^{\prime}-J_{3}{ }^{\prime \prime}\right) i\left(J^{\prime}\left|T_{\mu 4}\right| J^{\prime \prime}\right) .
$$

15 Ein ähnliches Ergebnis ist durch B. Podolsky u. C. Kik u chi (Physic. Rev. 65, 228 und 67, 184 [1944]) gefunden worden bei einem Problem, bei dem die L a g r a n g e-Funktion die zweiten Ableitungen der Wellenfunktionen nach der Zeit enthält. 
Aus der Wellengleichung (51) folgt, daß die rechte Seite von (53) sich wie eine $\delta$-Funktion in Abhängigkeit von $\left(J_{4}{ }^{\prime}-J_{4}{ }^{\prime \prime}\right)$ verhält.

Interessanterweise genügt Gl. (53) allein bereits, um das ganze Problem festzulegen. Wenn es gelingt, eine hermitesche Matrix $\left(J^{\prime}|\varphi| J^{\prime \prime}\right)$ zu finden, die Gl. (53) befriedigt, so befriedigt sie auch Gl. (51) wegen der nichtdiagonalen Glieder in (53), und die Vertauschungsrelationen (48) gelten dann wegen der Definitionen (49). Im ersten Augenblick scheint es merkwürdig, daß die Gl. (53) allein für die Gültigkeit nicht nur der Wellengleichung, sondern indirekt auch der Vertauschungsrelationen sorgen soll. Aber einen ähnlichen Sachverhalt findet man auch in der gewöhnlichen Quantenmechanik, obwohl er dort offenbar bisher noch nicht erwähnt worden ist. Um dies zu zeigen, betrachten wir ein System von einem Freiheitsgrad und definieren die Matrix $\dot{q}$ in Analogie zu (48) durch

$$
\left(E_{r}|\dot{q}| E_{s}\right)=\frac{i}{\hbar}\left(E_{r}-E_{s}\right) q_{r s} .
$$

Die Matrixgleichung für die Energie:

$$
E_{r} \delta_{r s}=\left(r\left|\frac{m}{2} \dot{q}^{2}\right| s\right)+(r|V(q)| s)
$$

sichert dann die Gültigkeit nicht nur der Bewegungsgleichung, sondern auch der Vertauschungsrelation. Um dies zu beweisen, vertauschen wir (55) mit $q$, und es ergibt sich

$$
\dot{q}=\frac{i}{\hbar} \frac{m}{2}\left(\dot{q}^{2} q-q \dot{q}^{2}\right)
$$

oder, indem man

setzt:

$$
\alpha=\frac{i}{\hbar} m(\dot{q} q-q \dot{q})
$$

$$
\dot{q}=\frac{1}{2}(\dot{q} \alpha+\alpha \dot{q})
$$

Die einzige* Lösung von (58) ist

$$
a=1 \text {, }
$$

was unmittelbar zur Vertauschungsrelation

$$
p q-q p=-i \hbar
$$

führt. Deshalb wird jede hermitesche Matrix $q$, die die Energiegleichung befriedigt:

* Anm. bei der Korrektur. Die Eindeutigkeit dieser Lösung hängt von gewissen physikalischen Forderungen $a b$, die einer näheren Untersuchung bedürfen und an einer anderen Stelle erörtert werden sollen.

$$
\begin{array}{r}
E_{r} \delta_{r s}=-\frac{m}{2 \hbar^{2}} q_{r l} q_{l s}\left(E_{r}-E_{l}\right)\left(E_{l}-E_{s}\right) \\
+\left(r\left|V_{(q)}\right| s\right),
\end{array}
$$

auch von selbst die Bewegungsgleichung und die Vertauschungsrelation (60) befriedigen, wenn $\dot{q}$ durch (54) definiert ist.

Die Gl. (49) und (53) legen die Quantisierung einer Wellengleichung von dem allgemeinen $\mathrm{Ty}$ pus (42) vollständig fest. Man kann deshalb zur nächsten Frage übergehen, wie die Matrix $S$ in einem solchen Formalismus bestimmt wird, wenn die Wellengleichung gegeben ist.

c) $\mathrm{Zu}$ diesem $\mathrm{Z}$ wecke ist es nützlich, den Ausdruck (53) für Energie und Impuls in zwei Teile einzuteilen, die als kinetische Energie (oder Impuls) der primären Teilchen und als Wechselwirkungsenergie (bzw. Impuls) bezeichnet werden können. Es ist zweifelhaft, ob diese Einteilung eindeutig ist. In vielen Fällen kann das erste Glied in (53), das den Faktor $A$ enthält, als kinetische Energie betrachtet werden. Dies würde bedeuten, daß die Nullstellen der Funktion $A(k)$ die Massen der Elementarteilchen bestimmen ${ }^{16}$, während die Ausdrücke mit den höheren Potenzen von $\varphi$ keine Rolle spielen, wenn die Teilchen weit voneinander entfernt sind. Aber es mag auch sein, daß einige Teile der letzteren Ausdrücke zum ersten Glied hinzugenommen werden müssen, um $\mathrm{zu}$ erreichen, daß der Rest bei weiten Abständen der Teilchen nichts beiträgt. Schließlich kann man auch einfach den niedrigsten Eigenwert $x$ für die Länge des Vektors $J$ bestimmen, der als die Masse des leichtesten Elementarteilchens bezeichnet werden kann; dann kann man in (52) einen Ausdruck mit $A(k)=\sum_{\mu} k_{\mu}{ }^{2}+\varkappa^{2}:$

$$
\begin{aligned}
\left.\int_{\mu}\left(J_{\mu}{ }^{\prime}-J_{\mu}{ }^{\prime \prime \prime}\right)^{2}+{ }^{2}\right\} & \left(J^{\prime}|\varphi| J^{\prime \prime \prime}\right)\left(J^{\prime \prime \prime}|\varphi| J^{\prime \prime}\right) \\
& \frac{\left(J_{\mu}{ }^{\prime \prime \prime}-J_{\mu}{ }^{\prime}\right)\left(J_{\nu}^{\prime \prime \prime}-J_{\nu}{ }^{\prime}\right)}{\left(J_{\lambda}{ }^{\prime \prime \prime}-J_{\lambda}{ }^{\prime}\right)\left(J_{\lambda}{ }^{\prime \prime}-J_{\lambda}{ }^{\prime}\right)}
\end{aligned}
$$

addieren und subtrahieren.

Dieser Ausdruck kann als kinetische Energie genommen werden, der Rest [(52) minus diesen Ausdruck] als Wechselwirkungsenergie; alle anderen Teilchen würden dann als zusammengesetztes System erscheinen, das aus mehreren Elementarteilchen besteht. Es ist ein interessanter Zug der Gln. (52) und (53), daß sie im allgemeinen

\footnotetext{
${ }^{16}$ Vergl. z. B. F. B o p p ${ }^{14}$.
} 
keine klare Unterscheidung zwischen Elementarteilchen und zusammengesetzten Systemen zulassen.

Wenn eine Matrix $\left(J^{\prime}|\varphi| J^{\prime \prime}\right)$ gefunden ist, die Gleichung (53) befriedigt, so wird es also auf jeden Fall möglich sein, die Matrizen für die kinetische Energie (bzw. den Impuls) zu berechnen. Die Eigenwerte dieser Matrizen können in der üblichen Weise durch Energie und Impuls der Elementarteilchen charakterisiert werden. Man kann also von der Matrixdarstellung, in $\operatorname{der} J_{\mu}$ diagonal ist, zu einer anderen Darstellung transformieren, in welcher der Vierervektor der kinetischen Energie und des Impulses diagonal ist. Die Transformationsfunktion entspricht der Lösung $\psi$ der Schrödinger-Gleichung (11). Man kann in der Tat $J_{\mu}$ in dieser neuen Matrixdarstellung ausdrücken und erhält auf diese Weise die $\mathrm{H}$ a milton-Funktion als eine Matrix in den Impulsen der Elementarteilchen.

Daher führt selbst die weite Verallgemeinerung der zugrundeliegenden Wellengleichungen zu Gleichungen von der Art (42) nicht zu einer allgemeineren Hamilton-Funktion als (10) oder (11) ; nur kann die Matrix der Wechselwirkungsenergie $\left(\mathfrak{f}^{\prime \prime}|V| \mathfrak{f}^{\prime}\right)$ in so komplizierter Weise von den Impulsen abhängen, daß die transformierte Matrix $\left(x^{\prime \prime}|V| x^{\prime}\right)$ nicht diagonal ist.

d) Es ist nicht bekannt, ob ein Formalismus von der Art (53) widerspruchsfrei durchgeführt werden kann, abgesehen von dem trivialen Fall $B=C=\ldots=0$. Die relativistischen $\mathrm{H}$ a milt on-Funktionen, die bisher untersucht worden sind und die Spezialfälle von Gl. (53) sind, führen sicher nicht $\mathrm{zu}$ widerspruchsfreien Ergebnissen. Aber selbst wenn eine bestimmte Klasse von Funktionen bekannt wäre, die zu widerspruchsfreien Ergebnissen führt, so würde diese Tatsache nicht wesentlich das Problem erleichtern, die richtige Hamilton-Funktion und damit die richtige $S$-Matrix zu finden; denn es besteht ja kein Grund dafür, daß die Hamil t on - Funktion eine einfache Form haben sollte, und die Invarianzbedingungen würden wohl kaum genügen, die richtige Form aus allen möglichen Formen auszuwählen.

Man kann aber das Problem der Elementarteilchen auch auf ganz andere Weise angreifen und versuchen, die Matrix $S$ unmittelbar zu bestimmen, ohne die Benutzung einer $\mathrm{Hamilton}$ Funktion. Es kann sein, daß die zukünftige Theorie überhaupt nicht mit Begriffen wie Wel- lengleichung oder Schrödinger-Funktion ar beitet, selbst nicht in der verallgemeinerten.Form (42) und (52). In diesem Fall muß die Matrix $S$ durch Beziehungen anderer Art bestimmt werden als in den Gln. (6) bis (18). Das einfachste Verfahren würde etwa darin bestehen, mit einer hermiteschen Matrix $\eta$ anzufangen, die relativistisch invariant ist und die mit Hilfe der Gl. $S=e^{i} \eta \mathbf{z u}$ einer unitären Matrix $S$ führt, die die Experimente richtig beschreibt. Die Matrix $\eta$ müßte dabei, ähnlich wie vorher die H a mil t o n - Funktion, nach Korrespondenz- und Invarianzgesichtspunkten gefunden werden.

Um diese Möglichkeit zu untersuchen, hat der Verfasser einige einfache Annahmen für $\eta$ und die zugehörigen Folgerungen für $S$ und für die beobachtbaren physikalischen Größen durchgerechnet. Die Ergebnisse mögen hier erwähnt werden:

Wir beginnen mit einer skalaren Funktion $\varphi$ mit der F o u rie r - Entwicklung

$\varphi=\Sigma\left(2 k^{0} V\right)^{-1 / 2}\left(a_{\mathfrak{p}} e^{i\left(\mathfrak{t} \mathfrak{r}-k^{0} t\right)}+a_{-} e^{i\left(\mathfrak{l} \mathfrak{r}+k^{0} t\right)}\right)$,

wobei $V$ das Normierungsvolumen bedeutet und wobei die Amplituden $a$ mit der Anzahl $N$ der Teilchen des Impulses $\mathfrak{f}\left(k^{0^{2}}=\varkappa^{2}+k^{2}\right)$ durch die Gleichungen

$$
\begin{aligned}
a_{\mathrm{t}}^{*} a_{\mathrm{p}}=N_{\mathrm{p}} ; a_{\mathrm{p}} N_{\mathrm{p}} & =\left(N_{\mathrm{p}}+1\right) a_{\mathrm{p}} ; \\
a_{\mathrm{p}}^{*} N_{\mathrm{p}} & =\left(N_{\mathrm{p}}-1\right) a_{\mathrm{p}}^{*}
\end{aligned}
$$

verbunden sind. Im Grenzfall eines unendlichen Volumens $V$ geht die Summe über $\mathfrak{t}$ über in ein Integral

$$
\sum_{i} \rightarrow \frac{V}{(2 \pi)^{3}} \int d^{k}
$$

Die einfachste Annahme für $\eta$, die zur Streuung von Elementarteilchen führt, lautet dann:

$$
\eta=\varepsilon \int d \mathfrak{r} d t \varphi^{4}
$$

wobei $\varepsilon$ eine numerische Konstante ist. Wenn man die Fourier-Entwicklung (62) in (65) einsetzt, erhält man Produkte von vier Faktoren $a_{\mathrm{f}}$ und $a_{e}{ }^{*}$. Nach J o r d a n und Klein wollen wir (65) durch die Annahme abändern, daß in diesen Produkten alle Faktoren $a_{f}{ }^{*}$ links von allen Faktoren $a_{\ell}$ stehen sollen. Diese Vertauschung ändert nichts an der relativistischen Invarianz von $\eta$. Wenn nur zwei Teilchen vorhanden sind, können die Matrixelemente $\eta_{i}$ in der Form 


$$
\left(\mathfrak{k}_{1}^{\prime \prime} \mathfrak{k}_{2}^{\prime \prime}|\eta| \mathfrak{k}_{1}{ }^{\prime} \mathfrak{k}_{2}{ }^{\prime}\right)=\frac{3 \varepsilon}{(2 \pi)^{2}}\left(k_{1}{ }^{0 \prime} k_{2}{ }^{0 \prime} k_{1}{ }^{0 \prime \prime} k_{2}{ }^{0 \prime \prime}\right)^{-1 / 2} \delta\left(\mathfrak{k}_{1}{ }^{\prime}+\mathfrak{k}_{2}{ }^{\prime}-\mathfrak{k}_{1}{ }^{\prime \prime}-\mathfrak{f}_{2}{ }^{\prime \prime}\right) \delta\left(k_{1}{ }^{0 \prime}+\ldots\right)
$$

geschrieben werden. Bringt man $\eta$ auf Diagonalform, indem man von $\mathfrak{f}$ auf die Kugelfunktionen der Richtung von $\mathfrak{t}$ übergeht, so findet man, daß nur die Wellen mit der azimutalen Quantenzahl $l=0$ (die mit zentraler Symmetrie) gestreut werden. Der Wirkungsquerschnitt für Streuung wird dann

$$
Q=\frac{4 \pi}{k^{2}} \sin ^{2} \frac{3 \varepsilon v}{8 \pi}
$$

$$
\begin{aligned}
& \frac{1}{\pi}\left[1-\frac{4 \varkappa^{2}}{\left(k_{1}{ }^{0 \prime}+k_{2}{ }^{\prime \prime}\right)^{2}-\left(\mathfrak{k}_{1}\right.}\right. \\
& \text { t, so findet man für den } \\
& Q=\frac{4 \pi \varepsilon^{2}}{k^{02}+\varepsilon^{2} k^{2}}
\end{aligned}
$$$$
\operatorname{arctg}\left(\varepsilon \sqrt{1-\frac{4 x^{2}}{\left(k_{1}{ }^{0 \prime}+k_{2}{ }^{0 \prime}\right)^{2}-\left(\mathfrak{k}_{1}{ }^{\prime}+\mathfrak{F}_{2}{ }^{\prime}\right)^{2}}}\right)
$$

•rsetzt, so findet man für den Wirkungsquerschnitt

wo sich wieder $k$ auf das Schwerpunktsystem bezieht. In dem Bezugssystem, in dem ursprünglich eines der Teilchen ruht, etwa $\mathfrak{f}_{2}{ }_{2}=0$, führt (69) zu dem Wirkungsquerschnitt

$$
Q=\frac{4 \pi \varepsilon^{2}}{x^{2}+\left(1+\varepsilon^{2}\right) \frac{\varkappa}{2}\left(k_{1}{ }^{0 \prime}-\varkappa\right)} .
$$

Der Eigenwert von $\eta$ für die kugelsymmetrische Welle $(l=0)$ ist:

$$
\eta_{l=o}=2 \operatorname{arctg}\left(\varepsilon \frac{k}{k^{0}}\right) \text {. }
$$

Diese Phase wird logarithmisch unendlich an dem Punkt

$$
\varepsilon k / k^{0}=i .
$$

Daher definiert (72) einen diskreten stationären Zustand des Systems. Zwei Teilchen können ein abgeschlossenes System bilden, wenn $\varepsilon$ negativ ist und $k$ negativ imaginär ist und der Gl. (72) genügt; die gesamte Ruhenergie $x_{2}$ dieses Systems ergibt sich nach (72) zu

$$
\varkappa_{2}=2 \varkappa|\varepsilon| i \sqrt{1+\varepsilon^{2}} .
$$

Der Ausdruck (68) liefert also ein Beispiel, bei dem zwei Teilchen sich in solcher Weise anziehen, daß sie in einem diskreten stationären $\mathrm{Zu}$ stand aneinander gebunden werden können; alle wo $v=k / k^{0}$ die Geschwindigkeit und $k$ der Impuls der Teilchen in dem Koordinatensystem ist, in dem der Schwerpunkt ruht. Die Annahme (65) entspricht daher der Annahme einer Kraft zwischen zwei Teilchen, die nur dann wirkt, wenn sich die beiden Teilchen am gleichen Punkt befinden.

Wenn man in Gl. (66) den Faktor $3 \varepsilon /(2 \pi)^{2}$ durch den komplizierteren Faktor anderen Zustände gehören zum gewöhnlichen kontinuierlichen Spektrum.

Schließlich ist eine Wechselwirkung untersucht worden, die zur Entstehung von neuen Teilchen führt. Wenn man

$$
\eta=\lambda^{2} \int d \mathfrak{r} d t \varphi^{6}
$$

setzt, so führt man dadurch Matrix-Elemente ein. die sechs Faktoren $a_{\mathrm{f}}{ }^{*}$ oder $a_{\mathrm{f}}$ enthaiten. Diese Faktoren geben Anlaß zu Übergängen, bei denen ein Zusammenstoß zwischen zwei Teilchen zur Schaffung eines neuen Paares von Teilchen führen kann. Die Wahrscheinlichkeit für Paarerzeugung bei gegebenen Anfangsbedingungen kann berechnet werden. Wenn die Gesamtenergie für die Erzeugung einer großen Anzahl von Teilchen genügt, so können Vielfachprozesse vorkommen, bei denen der Zusammenstoß energiereicher Teilchen zur Entstehung vieler Paare in einem explosionsartigen Akt führt. Das Auftreten solcher Vielfachprozesse hängt jedoch von den Einzelheiten der Wechselwirkungsmatrix (74) ab. Bei der genauen Form (74) treten solche Prozesse nicht ein; wenn man aber die Zahlenkoeffizienten in der binomischen Entwicklung von $\varphi^{6}[\varphi$ ist dabei durch (62) gegeben] ändert, so kommt man zu Wechselwirkungsmatrizen, die Vielfachprozesse zur Folge haben; für die Einzelheiten dieser Ergebnisse verweisen wir auf die Arbeit 1.c. ${ }^{3}$, II.

Wenn man in dieser Weise $S$ aus einer gegebenen Matrix $\eta$ bestimmt, so führt der Formalismus zu keinerlei Divergenzschwierigkeiten, und die Resultate befriedigen alle Forderungen der Relativitätstheorie und der Quantentheorie 
Daher ist ein solcher Formalismus befriedigender als die übliche Methode, bei der man mit einer $\mathrm{Hamilt}$ on schen Funktion beginnt, die sicher zu divergenten Resultaten führt. In der Tat kann man sogar sagen, daß die übliche Methode nur einen Sinn erhält, wenn man sie mit Hilfe des $\eta$-Formalismus deutet. Denn das gewöhnliche Verfahren benützt die sogenannte erste Näherung für den Vergleich mit den Experimenten. Dies kann nur bedeuten, daß die erste Näherung dazu verwendet werden soll, eine $\eta$-Matrix festzulegen, die dann $S$ für den Vergleich mit dem Experiment bestimmt. Ein unmittelbarer Vergleich der ersten Näherung mit den Experimenten wäre sinnlos, da die höheren Approximationen viel größere Beiträge geben würden; aber natürlich ergeben sich dabei keine anderen Resultate, als wenn man zuerst die $\eta$-Matrix und dann $S$ bestimmt. Auch abgesehen von diesem Argument sieht man nicht recht, warum es leichter sein sollte, die richtige $\mathrm{Ha}$ ilton-Funktion als die richtige $\eta$-Matrix eines gegebenen Systems zu finden; denn es gibt wohl keinen Grund dafür, daß in der Theorie der Elementarteilchen die $\mathrm{H}$ a milt on sche Funktion - wenn sie überhaupt existiert - einfacher sein sollte als die $\eta$-Matrix.

Allerdings gibt es einen anderen Grund für die Annahme, daß die zukünftige Theorie wahrscheinlich nicht mit einer einfachen $\eta$-Matrix beginnen kann. Irgendein einfacher Ausdruck für $\eta$ wie (65) oder (68) oder (74) scheint nämlich nicht in der gleichen Weise eine ,glatte“ Funktion der Impulse für die Matrix-Elemente zu ergeben wie eine $\eta$ - oder S-Matrix, die aus einer HamiltonFunktion abgeleitet ist. Es wäre eine sorgfältige Untersuchung nötig, zu entscheiden, welche Bedingungen von Glattheit eine $\eta$-Matrix erfüllt, die aus einem Formalismus wie (6) bis (18) folgt. Die $\eta$-Matrix (74) führt z. B. zu Diskontinuitäten bei den Werten der Gesamtenergie, die ein ganzzahliges Vielfaches der Ruhemasse der Teilchen sind; denn wenn man diese Werte überschreitet, werden neue Prozesse möglich, bei denen eine größere Anzahl von Teilchen erzeugt werden kann. Offenbar müssen diese Werte in einer gewissen Weise singulär sein. Aber es sieht so aus, als seien die $\eta$ - und $S$-Matrizen, die aus einer $\mathrm{Ha}$ milto $\mathrm{n}$ - Funktion stammen, glatter in der Umgebung dieser Punkte als der Ausdruck (74).

Dieses Argument weist in Richtung auf einen zukünftigen Formalismus, der bis zu einem ge- wissen Grade den Gln. (6) bis (18) ähnlich ist und glatte Funktionen für $S$ ergibt, der jedoch die Divergenzschwierigkeiten des gewöhnlichen Formalismus vermeidet. In dieser zukünftigen Theorie dürfte die universelle Länge von der Größenordnung $10^{-13} \mathrm{~cm}$ eine wichtige Rolle spielen, und man kann annehmen, daß der Formalismus in den gewöhnlichen übergeht an der Grenze, an der die universelle Länge als sehr klein angesehen werden kann - in ähnlicher Weise wie die Quantenmechanik in die klassische Mechanik übergeht, wenn $\hbar$ als klein betrachtet werden kann.

III. Vergleich mit dem gew öhnlichen Verfahren

a) Wenn es sich herausstellen sollte, daß es in der richtigen Theorie der Elementarteilchen keine Hamilt,on-Funktion gibt, so muß die physikalische Deutung des neuen Formalismus mit der des älteren verglichen werden. Ein wesentlicher Zug der Quantenmechanik ist die Existenz einer Wahrscheinlichkeitsfunktion, deren Quadrat die Wahrscheinlichkeit dafür angibt, ein Teilchen an einem gegebenen Punkt zu finden. Die Wahrscheinlichkeitsfunktion kann durch Experimente geprüft werden. Z. B. kann man durch Stöße anderer energiereicher Teilchen auf das zu prüfende Teilchen die Wahrscheinlichkeitsverteilung des zu prüfenden Teilchens vor dem Stoß ausmessen. Es ist ein wesentlicher Zug der Quantenmechanik, daß man bei solchen Versuchen stets die gleiche Wahrscheinlichkeitsverteilung erhalten muß, gleichgültig, ob man etwa ein $\gamma$-Strahl-Mikroskop oder ein Elektronenmikroskop oder irgendeine andere Teilchensorte verwendet.

Im neuen Formalismus kommt jedoch vielleicht der Begriff der Wahrscheinlichkeitsfunktion nicht vor; die Wahrscheinlichkeit für die Streuung eines energiereichen Teilchens durch das zu prüfende Teilchen wird trotzdem durch die Matrix $S$ bestimmt. Formal könnte man aus der Verteilung der gestreuten Teilchen eine Dichteverteilung des zu prüfenden Teilchens vor dem Stoß berechnen. Aber selbst wenn man davon absieht, daß diese Rechnung durch die physikalischen Gesetze nicht gerechtfertigt werden könnte, würde das Ergebnis der Rechnung wahrscheinlich von der Art der Teilchen abhängen, die für den Versuch verwendet werden. Das $\gamma$-Strahl-Mikroskop und das Elektronenmikroskop würden wahrscheinlich verschiedene Resultate ergeben, wenn 
es sich um Wahrscheinlichkeitsverteilungen innerhalb von Gebieten der Größenordnung $10^{-13} \mathrm{~cm}$ handelt. Nur für größere Gebiete, in denen der neue Formalismus in den früheren übergeht, würden die beiden verschiedenen Instrumente näherungsweise die gleiche Verteilung liefern.

Es muß untersucht werden, ob ein solches Verhalten unserer allgemeinen Kenntnis der Elementarteilchen entspricht. In der gewöhnlichen Quantentheorie würde man annehmen, daß verschiedene Instrumente die gleiche Dichteverteilung liefern, weil die Größe der Teilchen, verglichen mit der Ausdehnung der Dichteverteilung, sehr gering ist. Wenn man jedoch zu Gebieten von der Größenordnung $10^{-13} \mathrm{~cm}$ kommt, so sind diese Gebiete wahrscheinlich nicht größer als die Elementarteilchen selbst. Natürlich ist es schwierig, den Durchmesser eines Elementarteilchens überhaupt zu definieren. Aber wenn die universelle Länge von der Größenordnung $10^{-13} \mathrm{~cm}$ eine Rolle in der Theorie spielt, so ist es vernünftig, sich die Elementarteilchen als Gebilde vorzustellen, die über ein Gebiet dieser Größenordnung ausgebreitet sind. Es dürfte auch kein reiner $\mathrm{Zu}$ fall sein, daß die Compton sche Wellenlänge der schwersten Elementarteilchen die gleiche Größenordnung $10^{-13} \mathrm{~cm}$ hat und daß alle schwereren Teilchen aus Neutronen und Protonen zusammengesetzte Systeme mit größerer Ausdehnung zu sein scheinen (z.B. die Atomkerne irgendeines schweren Elements). Es ist deshalb ganz natürlich, daß man aus den Messungen nicht in eindeutiger Weise eine Dichteverteilung innerhalb eines Gebietes von $10^{-13} \mathrm{~cm}$ erschließen kann.

b) Der in den Gln. (9) und (21) festgelegte mathematische Rahmen für eine zukünftige Theorie der Elementarteilchen mag noch verglichen werden mit den früheren Versuchen, ein vollständiges mathematisches Schema für die Quantenelektrodynamik oder für die Yukawa-Feldtheorie anzugeben. Zunächst ist hier zu beachten, daß die Gln. (6) bis (18) etwas abgeändert werden müssen, wenn es sich um geladene Teilchen handeln soll, die aufeinander durch Kräfte langer Reichweite wirken. Bekanntlich muß bei einer einfallenden oder auslaufenden Kugelwelle der Ausdruck $\frac{1}{r} \mathrm{e}^{i k r}$ im Falle der Cou lo m b-Kraft durch den Ausdruck

$$
\frac{1}{r} \exp \left[ \pm i\left(k r-\frac{k^{0} \cdot e^{2}}{k} \lg r\right)\right]
$$

ersetzt werden; für den Impulsraum bedeutet dies, daß die singulären Funktionen $\delta_{ \pm}\left(k^{0^{\prime}}-k^{0^{\prime \prime}}\right)$ in Gl. (6) durch Ausdrücke der Art

$$
\frac{1}{2 \pi i}\left[ \pm\left(k_{0}{ }^{\prime}-k_{0}{ }^{\prime \prime}\right)\right]^{ \pm i e^{2} k^{0} / \hbar c k^{\prime}-1}
$$

ersetzt werden müssen. Abgesehen von dieser Änderung kann man wieder beweisen, daß $S^{\dagger} S=1$ ist, und die früheren Rechnungen können im wesentlichen übernommen werden.

Der gewöhnliche Formalismus der Quantenelektrodynamik beginnt mit der bekannten Hamiltonschen Funktion und betrachtet die Wechselwirkung zwischen Elektronen und $\mathrm{Max}$ wellschem Feld als eine kleine Störung. Wenn man die Störungsenergie zweiter Ordnung berechnet, so erhält man eine Selbstenergie des Elektrons, die logarithmisch divergiert ${ }^{17}$. Man kann jedoch diese divergierenden Glieder durch ein Subtraktionsverfahren ${ }^{18}$ abziehen und dann zu höheren Näherungen übergehen. Vom Gesichtspunkt des $\eta$-Formalismus aus kann man dieses Verfahren in der folgenden Weise beschreiben: Indem man in der üblichen Weise durch eine Störungsrechnung die Wahrscheinlichkeitsamplituden für die Streuung und die Ausstrahlung von Licht usw. bestimmt, erhält man eine gewisse erste Näherung der $\eta$-Matrix. Diese $\eta$-Matrix erster Ordnung beschreibt die Experimente innerhalb dieser Näherung richtig und gibt außerdem widerspruchsfreie Resultate in jeder beliebigen höheren Näherung, da der $\eta$-Formalismus nicht zu Divergenzen führt. Man kann aber auch die Störungsrechnung einen oder mehrere Schritte weiter verfolgen, wobei man jeweils die divergenten Glieder abzuziehen hat, und man erhält in dieser Weise Änderungen von $\eta$ in höherer Ordnung. Diese modifizierte $\eta$-Matrix gibt auch widerspruchsfreie Ergebnisse in- jeder beliebigen Näherung, und die so gewonnenen Resultate werden sich im allgemeinen ein wenig von denen unterscheiden, die aus der erstgenannten $\eta$-Matrix gewonnen waren. Es dürfte aber eine offene Frage sein, ob die modifizierte $\eta$-Matrix die Experimente besser darstellt als die $\eta$-Matrix erster Näherung. Denn in beiden Fällen wird die $\eta$-Matrix korrespondenzmäßig der klassischen Elektrodynamik entsprechen, und beide $\eta$-Matrizen geben wider-

17 V. We i n k o p f, Z. Physik 89, 27 u. 90, 817 [1934].

18 P. A. M. D ir a c, Bakerian lecture, l. c. ${ }^{1}$; W. P a u l i , Rev. mod. Physics [1943]. 
spruchsfreie Resultate. Aus dieser Überlegung erkennt man, daß die übliche Forderung der Korrespondenz zwischen Quantentheorie und klassischer Theorie sicher nicht ausreicht, um den quantentheoretischen Formalismus festzulegen.

Noch offenbarer ist die Mehrdeutigkeit des üblichen Formalismus in der Theorie des $\mathrm{Yu}$ kawa-Feldes. Man kann hier entweder die Tatsache anerkennen, daß die Kupplung zwischen dem Yukawa-Feld und den schweren Teilchen stark ist ${ }^{19}$, wenn die Kernkräfte durch das Y ukawa-Feld erklärt werden sollen, oder man kann ein Subtraktionsverfahren anwenden, um die Glieder starker Kupplung zu beseitigen. Die beiden Möglichkeiten führen $\mathrm{zu}$ ganz verschiedenen $\eta$-Matrizen und daher auch $\mathrm{zu}$ ganz verschiedenen physikalischen Ergebnissen. Es dürfte einstweilen schwierig sein, zu entscheiden, welche Annahme den Experimenten besser entspricht. Vielleicht werden die qualitativen Züge beider Theorien in der späteren Theorie eine Rolle spielen.

19 G. W e n t z e l, l. c. ${ }^{2}$. c) Zum Schluß wollen wir versuchen, die Bedingungen zusammenfassend $\mathrm{zu}$ formulieren, die eine Theorie der Elementarteilchen erfüllen muß: Eine solche Theorie muß zu einer relativistisch invarianten unitären Matrix $S$ führen, die das asymptotische Verhalten der Wellen und die stationären Zustände in der üblichen Weise bestimmt. Der Formalismus, der $S$ festlegt, muß in der Grenze, in der die universelle Länge von der Größenordnung $10^{-13} \mathrm{~cm}$ als klein angesehen werden kann, in den üblichen Formalismus mit einer $\mathrm{H}$ amilton schen Funktion übergehen. Aber auch abgesehen von diesem Grenzfall, muß der neue Formalismus dem früheren insofern ähnlich sein, als er eine Funktion $S$ ergeben muß, die in derselben Weise „glatt" ist wie die aus gewöhnlichen Hamilton-Funktionen bestimmten $S$-Funktionen. Der richtige Formalismus liegt also wahrscheinlich irgendwo in der Mitte zwischen einem Formalismus der Art (42) und einem Schema, bei dem einfach die Matrix $\eta$ gegeben wird.

\title{
Zur Theorie des Elektrons
}

\author{
Von Walter Wessel, Graz ${ }^{1}$
}

(Z. Naturforschg. 1, 622-636 [1946]; eingegangen am 13. Juli 1946)

$I^{\prime}$ n dem Bestreben, die Reaktionskraft der Strahlung auf ein bewegtes, geladenes Teilchen in die Quantenmechanik einzubauen und so vielleicht den Schwierigkeiten der Quantenelektrodynamik zu entgehen, wurde der Verf. schon vor längerer Zeit $^{2}$ auf sehr merkwürdige Zusammenhänge zwischen Strahlungskraft und Spin geführt, die zunächst allen Deutungsversuchen widerstanden. Nach verschiedenen Bemühungen, der Sache von der klassischen ${ }^{3}$ oder der quantentheoretischen ${ }^{4}$ Seite nahezukommen, ließ sich zuletzt das Problem in eine klarere Form bringen durch die Fragestellung ${ }^{5}$, ob und welche Vertauschungsrelationen mit einem die Strahlungskraft enthaltenden System von Bewegungsgleichungen eines Elektrons vereinbar wären. Folgendes wurde erreicht:

1 z. Zt. in Heidelberg.

2 W. W e s s e l, Z. Physik 92, 407 [1934].

3 W. W e s s e l, Z. Physik 110, 625 [1938].
1. Man kann durch einen Kunstgriff von ungezwungener Einfachheit die von der bewegten Ladung ausgestrahlte Energie in der Ruhmasse mitzählen. Das System wird dann konservativ, und es lassen sich kanonische Impulse einführen. Wegen des Einflusses der Strahlungsreaktion wird der kanonische Impuls nicht nur Funktion der Geschwindigkeit, sondern auch der Beschleunigung. Impuls und Geschwindigkeit werden dadurch unabhängige Veränderliche. Diese Unabhängigkeit ist eine der wesentlichsten Eigentümlichkeiten der D i r a c schen Spintheorie.

2. Die Hamilt on sche Funktion nimmt genau die Diracsche Form an. Das ist möglich, weil darin auch bei D i r a c nur Koordinaten, Impulse und Geschwindigkeiten, dagegen keine Spinoperatoren auftreten. Da die Ruhmasse wegen der darin mitgezählten Strahlungsenergie zeitveränderlich

4 W. W e s s e l, Naturwiss. 30, 606 [1942].

5 W. W e s s e l, Ann. Physik (5) 43, 565 [1943]. 